\title{
Universidades para el Bienestar Benito Juárez García: un análisis socioespacial de su cobertura
}

\section{Universidades para el Bienestar Benito Juárez García: A Socio-Spatial Analysis of Coverage}

\author{
José Luis González Callejas (1) https://orcid.org/0000-0002-5201-9294 \\ Gustavo Mejía-Pérez (2) https://orcid.org/0000-0002-2370-4276 \\ Humberto González-Reyes (3) https://orcid.org/0000-0002-0677-3931 \\ (1) Universidad Autónoma Metropolitana \\ (2) Universidad Pedagógica Nacional \\ (3) Centro de Investigación y de Estudios Avanzados del Instituto Politécnico Nacional \\ (Recibido: 2 de enero de 2020; Aceptado para su publicación: 19 de junio de 2020)
}

Cómo citar: González, J. L., Mejía-Pérez, G. y González-Reyes, H. (2021). Universidades para el Bienestar Benito Juárez García: un análisis socioespacial de su cobertura. Revista Electrónica de Investigación Educativa, 23, e27, 1-15. https://doi.org/10.24320/redie.2021.23.e27.3733

\section{Resumen}

El objetivo del artículo es analizar desde una perspectiva socioespacial el cumplimiento de los criterios que el Programa de Universidades para el Bienestar Benito Juárez García definió para ubicar sus 100 primeras sedes a lo largo del territorio mexicano. En la primera parte se describe brevemente el programa; luego, mediante el software Quantum Geographic Information System, se analizaron las variables: población, pobreza, oferta de educación superior, migración, porcentaje de población indígena y violencia a nivel municipal. El estudio concluye que sólo uno de los planteles cumple con la totalidad de los criterios, lo que pone en duda su capacidad para ampliar las oportunidades de educación superior para personas que viven en municipios con altos niveles de marginación.

Palabras clave: educación superior, política educacional, oportunidades educacionales, acceso a la educación

\section{Abstract}

The objective of this article is to examine, from a socio-spatial perspective, the fulfillment of the criteria set by the Universidades para el Bienestar Benito Juárez García Program in establishing its 100 campuses throughout Mexico. First, we briefly describe the program, and then use the Quantum Geographic Information System to analyze the following variables: population, poverty, higher education programs offered, migration, percentage of indigenous population, and violence, at the municipal level. The study concludes that just one of the campuses meets the full set of criteria, casting doubt on the program's ability to open up higher education opportunities to individuals living in highly marginalized municipalities. 


\section{Introducción}

La expansión de la Educación Superior (ES) en México se ha desarrollado de manera similar a su desconcentración. De acuerdo con Didou (1997), durante los últimos decenios del siglo XX se ampliaron las redes de instituciones de educación superior (IES) y ciudades universitarias en torno a tres dinámicas: la promoción de municipios al rango de sede de un establecimiento universitario, la diferenciación interna de los establecimientos, y la ampliación de oferta de carreras en ciudades con al menos una IES. La expansión de las IES en el territorio nacional y la captación de sus alumnos se desarrollaron en un contexto de no competencia interinstitucional, lo que incrementó las tasas de atención en lugares donde las capacidades de respuesta a la demanda de inscripción eran mínimas o nulas.

El sector público incrementó su oferta desconcentrando las unidades de las universidades públicas estatales y creando sedes foráneas (Didou, 2011). Instituciones como la Universidad Nacional Autónoma de México, el Instituto Politécnico Nacional y la Universidad Autónoma Metropolitana abrieron planteles en entidades fuera de la Ciudad de México. Por su parte, universidades estatales como la Universidad Autónoma del Estado de México, la Universidad Autónoma del Estado de Hidalgo y la Universidad de Guadalajara ampliaron su cobertura en varias regiones de sus respectivas entidades. Además, surgieron nuevas IES públicas: universidades tecnológicas, politécnicas, interculturales y otras de apoyo solidario (Maldonado y Mejía, 2018). Paralelo al crecimiento de la oferta pública, el sector privado registró un incremento significativo en cuanto al número de establecimientos (Acosta, 2005; Silas, 2005).

Si bien estos procesos han acercado IES a quienes viven fuera de las grandes ciudades, deben hacerse precisiones regionales. Durante la década de 1990, entre el 50 y 60\% de la matrícula total en ES se concentró en seis entidades federativas: Distrito Federal, Estado de México, Jalisco, Puebla, Nuevo León y Veracruz (Márquez, 2004). Para 2007 la mitad de la matrícula se concentraba en siete estados, y 40 \% era atendida por sólo diez instituciones de ES (Poder Ejecutivo Federal, 2007, p. 179). Al respecto, Didou (2011) señala que la expansión de la ES ofreció a la población de escasos recursos un servicio educativo cuyo costo de accesibilidad se abarató por la disminución de la lejanía geográfica entre el domicilio y la institución; sin embargo, la oferta de formación que se ha creado aún es limitada en el número y en los perfiles de carreras. Además, la localización, diversidad y diferenciación de las instituciones de ES también reflejan diferentes niveles de acceso y de calidad (Brunner, 2005).

El crecimiento de la matrícula de ES en México ha sido particularmente notable durante la primera década de este siglo. Según reporta Mendoza (2015), la población estudiantil pasó de 2057249 en el año 2000, a 3449366 en 2012, lo que en términos de tasa bruta de cobertura significó pasar del 20.6 al 32.1\%. De acuerdo con la Secretaría de Educación Pública (SEP), para el ciclo escolar 2017-2018 la matrícula en ES en modalidad escolarizada y no escolarizada fue de 4561792 estudiantes, que representa una tasa bruta de cobertura de $38.4 \%$.

De acuerdo con Brunner et al. (2008), a inicios de este siglo el 45\% de jóvenes entre 18 y 24 años de clase media y media alta, provenientes de entornos urbanos en América Latina ingresó a la ES, mientras que sólo $3 \%$ de jóvenes provenientes de zonas rurales y marginadas logró hacerlo.

Рara el ciclo 2014-2015, la tasa neta de cobertura de ES en espacios rurales en México era de 9.5 y para espacios urbanos de 29.4\% (Socio-Economic Database for Latin America and the Caribbean y Banco Mundial, 2017). Así, aunque el número de escuelas, diversidad de programas, modalidades y cobertura territorial aumentó en los últimos 20 años, permanecen las diferencias de acceso en función de estratos socioeconómicos y poblaciones rurales y urbanas.

La condición socioeconómica continúa siendo uno de los factores más importantes que intervienen en la progresión escolar en México. En 2010 el porcentaje de los jóvenes del decil más alto de ingresos era casi siete veces mayor que la proporción que se ubicaba en el decil más pobre, estos datos muestran que la equidad es uno de los retos más importantes de la ES en México (Silva, 2014). 
En este contexto, el actual gobierno mexicano (2018-2024) puso en marcha el programa Universidades para el Bienestar Benito Juárez García (UBBJG). El primero de diciembre de 2018, el ejecutivo nacional anunció la creación de un nuevo programa educativo de nivel superior "gratuito y de alta calidad", que se enfocaría en hacer llegar la ES a las zonas más alejadas y marginadas del país.

Teniendo como marco este panorama, el presente trabajo analiza, desde una perspectiva socioespacial, en qué medida la localización de las sedes de las UBBJG cumple con los criterios establecidos en el programa y, en este sentido, explora en qué medida este programa social favorece el acceso de los jóvenes que viven en los municipios más pobres del país con poca o nula oferta de ES.

Los estudios relacionados con las UBBJG han sido escasos. El principal referente, hasta el momento, lo constituye una investigación realizada por la asociación Mexicanos Contra la Corrupción e Impunidad (MCCI) (2020). En ese estudio se evalúa la calidad de los servicios educativos del programa mediante un análisis de su diseño, su implementación y su enfoque de expansión, con base en el análisis de datos nacionales y visitas a 30 planteles de las UBBJG. El estudio encuentra que existen inconsistencias de diseño, carencias de infraestructura en los planteles, planta docente insuficiente y problemas para focalizar a la población objetivo.

En nuestra opinión, las principales limitaciones de esa investigación residen en que no incluye algunos criterios del diseño de las UBBJG, como son: el número de habitantes de la localidad, migración, proporción de población indígena y violencia, y que, consideramos, permitirían evaluar de forma integral los propios límites del programa. De igual forma, el citado estudio no incluye el análisis de las sedes ubicadas en la Ciudad de México y en el norte del país.

Por tanto, este trabajo -cuyos primeros resultados preceden a la publicación del estudio de MCClpretende realizar un análisis más amplio sobre las UBBJG, considerando cada una de las dimensiones que contempla su diseño. De esta manera se busca valorar, desde una perspectiva socioespacial, los alcances que el programa pudiera tener dentro de sus propios límites, y aportar evidencia que permita orientar el desarrollo de políticas enfocadas a la expansión de la oferta educativa en el nivel superior, especialmente aquellas dirigidas a poblaciones socialmente desfavorecidas.

Cabe aclarar que la información del artículo sólo corresponde a los primeros 100 planteles con que inició el programa, que actualmente cuenta con 145 sedes.

\subsection{El programa de las UBBJG: el proyecto}

El objetivo del programa UBBJG consiste en contribuir al bienestar social y la igualdad de la población más desfavorecida mediante la creación de 100 planteles en comunidades donde ya se imparte bachillerato y no existen opciones cercanas para cursar estudios superiores; o bien, sus programas educativos son poco pertinentes para las necesidades regionales. Los otros criterios para decidir la localización de las sedes son: municipios con altos índices de pobreza, población mayoritariamente indígena, un rango de entre 15 y 50 mil habitantes, población joven con problemas de drogadicción, delincuencia y altos índices de migración y de violencia (Organismo Coordinador de las Universidades para el Bienestar Benito Juárez García [OCUBBJG], 2020a; Roldán, 2019).

Las UBBJG ofrecen 36 carreras agrupadas en seis áreas de conocimiento. La de mayor presencia es Ingeniería Agroalimentaria, que se imparte en 17 planteles. Aunque en un principio se informó que se impartiría la licenciatura en Educación física con especialidad en beisbol (Carrera de beisbol..., 2019), hasta el momento esta carrera no se oferta en ningún plantel. Los programas educativos que se ofrecen son presenciales y tienen una duración de ocho ciclos escolares de 14 o 18 semanas cada uno (UBBJG, 2020). De acuerdo con declaraciones periodísticas, los títulos expedidos por las UBBJG tendrán validez en toda América Latina (Poy, 2018).

Dentro de las características de los servicios educativos de las UBBJG destaca que la modalidad será presencial e incluirá la realización de actividades independientes, uso de recursos en línea y apoyos 
tutoriales. Además, los estudiantes deben cumplir con el 85\% de asistencia, realizar todos los trabajos que les sean encomendados y acatar lo señalado en el reglamento escolar (OCUBBJG, 2020a).

De acuerdo con fuentes oficiales, cada uno de los planteles cuenta con espacios suficientes para el estudio: biblioteca, sala de cómputo, aulas espaciosas, comedor, laboratorios, así como campos de cultivo y espacio para el conocimiento y producción de energías renovables. Se espera que cada sede de las UBBJG atienda a 1600 alumnos. Durante el primer año de funcionamiento de la institución se busca matricular hasta 96 000 estudiantes y al finalizar el sexenio incorporar a 300000 jóvenes (SEP, 2019).

La administración de esta IES está a cargo del Organismo Coordinador de las Universidades para el Bienestar Benito Juárez García, entidad descentralizada de la SEP con facultad para determinar y aprobar sus planes y programas de estudio, así como de expedir títulos académicos, con sede en el municipio de Pátzcuaro (Michoacán) (DOF, 30 de julio de 2019). En 2019, el monto destinado para la edificación y operación de las UBBJG fue de mil millones de pesos; sin embargo, este monto se redujo para 2020, quedando en 987 millones (SHCP, 2019).

Hasta mediados de junio de 2019 su sitio web indicaba que sólo hay una carrera por municipio (con excepción del plantel de Zacapu (Michoacán), en el que se ofertan cuatro programas), y que los planes de estudio son originales y pertinentes para la atención de las necesidades de supervivencia, bienestar y sustentabilidad de la población. Este programa tiene la finalidad de que sus egresados obtengan de manera inmediata un lugar dónde trabajar y cuenten con la instrucción necesaria para atender la demanda en la comunidad en donde recibieron instrucción escolar (Gobierno de AMLO creará...., 2018).

Las UBBJG buscan formar profesionales con capacidad crítica y analítica, con altos estándares éticos y compromiso social que estén guiados por una vocación de servicio, ideales de justicia, dignidad y equidad, además de actuar conforme a valores como la humildad, solidaridad y entrega a los grupos sociales más necesitados, entre otras características (OCUBBJG, 2020a).

El ingreso a esta IES no contempla un examen de admisión, sino la valoración diagnóstica de los aspirantes para identificar sus antecedentes y necesidades de formación. Los aspirantes hacen un pre-registro y envían la documentación requerida por vía electrónica. En caso de que la demanda exceda la capacidad de un plantel se contempla realizar un sorteo para definir el ingreso (UBBJG, 2020). Los coordinadores del programa destacan que, a diferencia del resto de las instituciones públicas de educación superior, en las UBBJG todos los servicios son gratuitos, además de que los alumnos cuentan con una beca mensual de 2 400 pesos (en promedio, 120 dólares) (SEP, 2019) para por lo menos 15738 jóvenes de escasos recursos (Montes, 2018).

La distribución geográfica de los planteles no es homogénea; el estado de Oaxaca, por ejemplo, es la entidad que cuenta con el mayor número de planteles (11). De estas 11 sedes, 6 operaban desde 2016 como parte del Programa de Escuelas Universitarias, coordinado por el partido Movimiento Regeneración Nacional (MORENA), algunas de las cuales carecían de registro de validez oficial por parte de la SEP.

Las UBBJG iniciaron operaciones el 25 de marzo de 2019, y para finales del mismo mes se reportaba que estaban funcionando 51 de los 100 planteles. En la presentación del programa en el municipio de Aquismón (San Luis Potosí), Raquel Sosa, titular del Organismo Coordinador de las UBBJG, manifestó que para finales de abril todos los planteles estarían funcionando; señaló que los programas educativos estaban listos y que los planteles ya ofrecían 32000 espacios para jóvenes entre 18 y 29 años, aunque esta información sólo se pudo consultar hasta los últimos meses de 2019 (Gobierno de México, 2019).

En enero de 2020 se reportó que la totalidad de las sedes se encontraba operando, aunque se anunciaba el inicio de la construcción de 85 planteles, ya que varias de las sedes estaban desarrollando sus actividades en instalaciones prestadas. Además, se indicó que las UBBJG contaban con una matrícula de 42775 estudiantes y se esperaba que en 2021 se contaría con los primeros titulados (Salazar, 2020). 
La plantilla docente se eligió a partir de la postulación voluntaria de los candidatos, vía Internet, en febrero de 2019. Estos recibieron una primera capacitación durante dos días en las instalaciones del Centro de Cooperación Regional para la Educación de Adultos en América Latina y El Caribe (CREFAL); tras un proceso de selección se eligió a quienes fueron contratados, para, posteriormente, capacitarlos entre el 21 y el 22 de marzo (3 días antes de iniciar labores). El pago a los profesores es de 200 pesos la hora. Se prevé que la mayoría sea contratada por tiempo completo, lo que sumaría 9600 pesos mensuales, más las prestaciones de ley (Roldán, 2019).

\section{Método}

El presente estudio se realizó desde una perspectiva socioespacial y recuperó un par de supuestos de la geografía de la educación:

a) La variación en la provisión de oportunidades educativas y aprovechamiento escolar es un fenómeno social que implica problemas habitacionales y de salud, que pueden localizarse en la intersección entre el espacio, la estructura y los procesos sociales (Buttler y Hamnett, 2007).

b) Existe una distribución espacial de recursos y oportunidades educativas (Taylor, 2009).

Las UBBJG buscan atender personas que se ubican en contextos con características específicas, las cuales constituyen las variables de nuestro estudio: una población entre 15000 y 50000 habitantes, importantes niveles de pobreza, violencia, falta de oferta de ES, migración y altos porcentajes de población indígena.

Para identificar los municipios más pobres del país tomamos los datos del grado de marginación (Consejo Nacional de Población [CONAPO], 2015). Para la delimitación de las divisiones geopolíticas se tomó como referencia el Marco Geoestadístico 2017 del Instituto Nacional de Geografía y Estadística (INEGI). También se utilizó el Anuario Estadístico 2017-2018 de la Asociación Nacional de Universidades e Instituciones de Educación Superior (ANUIES, 2019), pues ofrece información sobre la oferta de ES pública y privada por municipio. Sólo se tomaron en cuenta las escuelas que ofrecen estudios de licenciatura, técnico superior universitario y educación normal en modalidad escolarizada. Finalmente, para localizar las sedes de las UBBJG se partió del listado que se indica en la información oficial (UBBJG, 2019).

Toda esta información se procesó en un Sistema de Información Geográfico (SIG), el software Quantum Geographic Information System (Q-GIS), que fue la herramienta con la que se desarrollaron los mapas que más adelante se presentan.

\section{Resultados}

Con base en lo anterior, este apartado presenta en qué medida se cumplen los criterios que el programa de las UBBJG estableció para la instalación de sus sedes.

\subsection{Población}

Consultamos la Encuesta Intercensal 2015 del INEGI para conocer el número de habitantes por municipio de aquellas localidades donde se ubican los planteles de las UBBJG. Ocho municipios se encuentran por debajo del mínimo poblacional establecido por el programa; mientras que 47 de ellos exceden el máximo estipulado. Sólo 52 casos cumplen con la condición de tener entre 15000 y 50000 habitantes; de modo que 48 de las sedes de las UBBJG no cumplen con el criterio poblacional.

La desproporción poblacional por municipio es considerable, pues si bien existen municipios como Áporo (Michoacán), con una población de 3218 habitantes, existen otros, como la alcaldía de Iztapalapa, en la Ciudad de México, con 1827868 personas. Así pues, no sólo se incumple este criterio en casi la mitad de las localidades donde se ubican los planteles; sino que el grado de la desproporción entre los mismos evidencia inconsistencias en el diseño del programa que podría generar problemas de focalización de la población objetivo. 


\subsection{Pobreza}

El grado de marginación es una medida que permite diferenciar entidades y municipios del país según el impacto de las carencias que padece la población. Cuanto mayor es el grado de marginación, más alta es la proporción de población juvenil y adulta en condiciones de aislamiento geográfico, con ingresos monetarios reducidos, carentes de una escolaridad mínima y con vivienda inadecuada. Los grados de marginación se clasifican en: muy alto, alto, medio, bajo y muy bajo (CONAPO, 2015).

Se usaron las coordenadas de geolocalización de los palacios municipales o alcaldías como referente espacial, pues en muchos casos aún no se cuenta con la dirección exacta de la ubicación de las sedes. ${ }^{1}$

En primer lugar, se identificaron los municipios de la República de acuerdo con el grado de marginación, así como las sedes de las UBBJG. La Figura 1 muestra este panorama.

Figura 1. Grado de marginación de los municipios de la República Mexicana y ubicación de sedes de las UBBJG

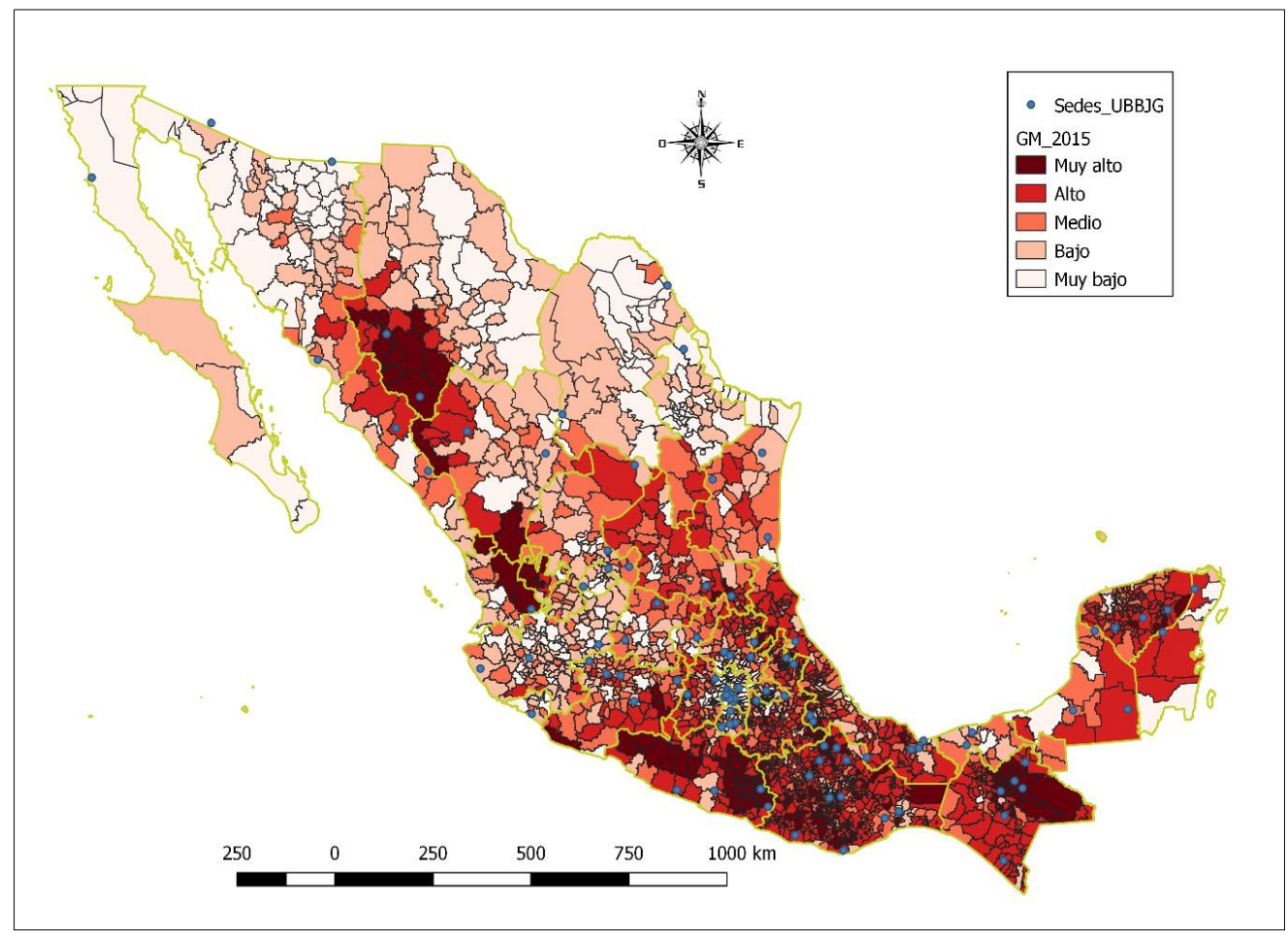

En la figura 1 se observa que entre más alto sea el grado de marginación de un municipio, más oscura será la tonalidad del color rojo. Resalta que los municipios más pobres tienden a estar juntos, creando "zonas de marginación" rodeadas de municipios con menor rezago. De acuerdo con datos del Consejo Nacional de Población (CONAPO, 2015) existen 2457 municipios en la República Mexicana, de los cuales 283 tienen muy alto grado de marginación, lo que representa el 11.5\% del total nacional. El panorama general de la ubicación de las sedes de las UBBJG muestra que estas escuelas se ubican en municipios pobres, con alto y muy alto grado de marginación; sin embargo, esta figura también muestra que en el centro del país hay una gran concentración de sedes de las UBBJG, donde el grado de marginación es muy bajo.

El acercamiento que presentamos de esta región en la Figura 2 nos permite destacar los matices entre las zonas de marginación y las zonas de abundancia en la parte central del Estado de México y de la Ciudad de México.

\footnotetext{
${ }^{1}$ En la sede de Urique (Chihuahua), se indica que el plantel de la UBBJG se ubicará en la comunidad de San Rafael.
} 
Figura 2. Grado de marginación de los municipios del centro la República Mexicana y ubicación de sedes de la UBBJG

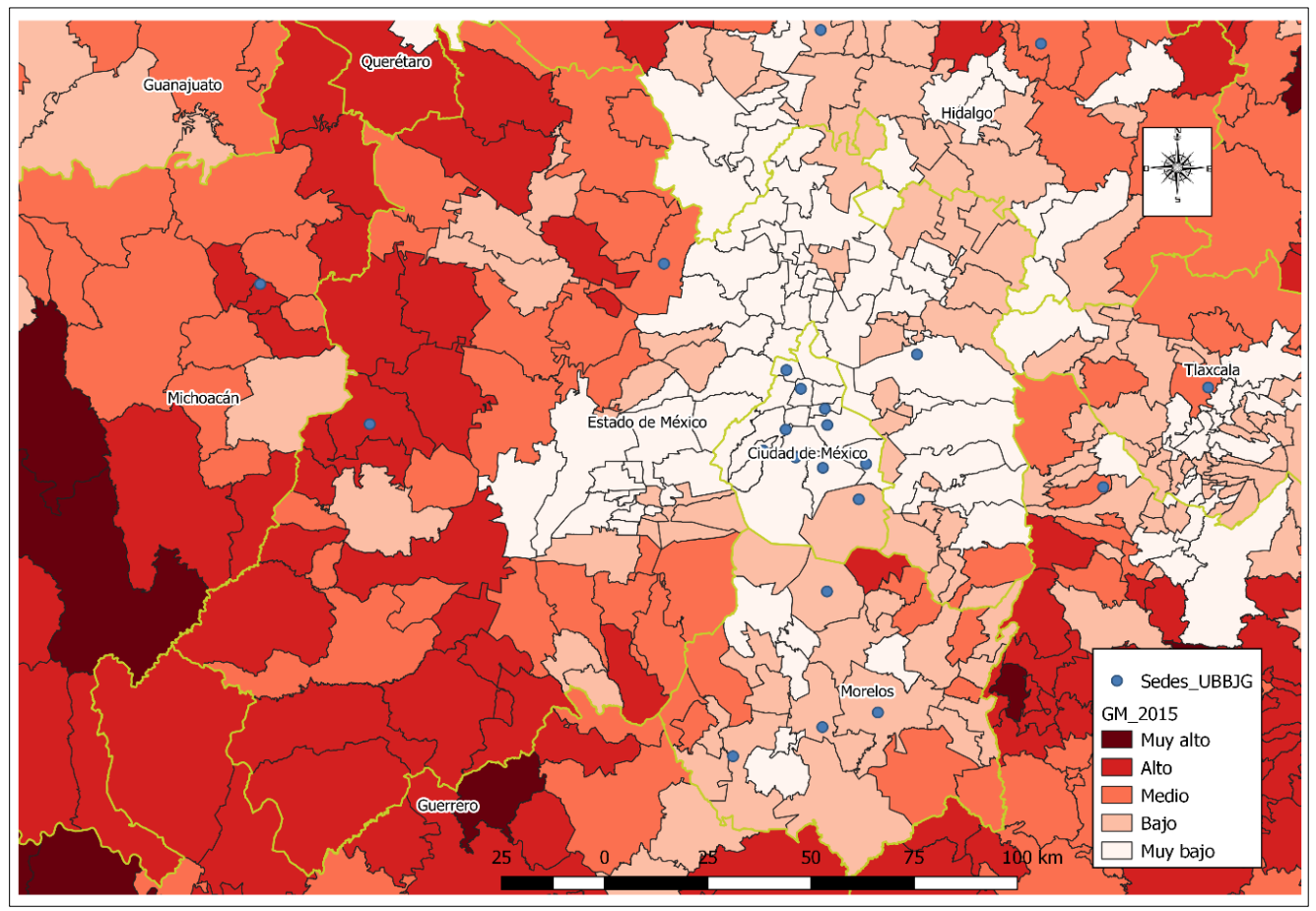

Otro detalle que se observa en la Figura 2 es que la tendencia en la ubicación de las sedes de las UBBJG que advertimos a nivel nacional cambia en esta región: las escuelas se ubican principalmente en municipios con grados de marginación muy bajo y bajo, y pocas en lugares con muy alto grado de marginación. Esta distribución no sólo indica el cumplimiento de los objetivos del programa UBBJG, sino que incluso los contradice, pues ubica algunos de sus planteles en localidades para los que no fue diseñado y que, como veremos a continuación, podría incluso agravar las condiciones de exclusión y marginación educativa si se contempla la variable oferta de ES.

\subsection{Oferta de educación superior}

Otra variable que se exploró fue la oferta de ES en los municipios donde se localizan las sedes de las UBBJG. La Figura 3 muestra el resultado del cruce entre el grado de marginación y la oferta de ES a nivel municipal. 
Figura 3. Sedes de las UBBJG por grado de marginación y oferta de educación superior de los municipios

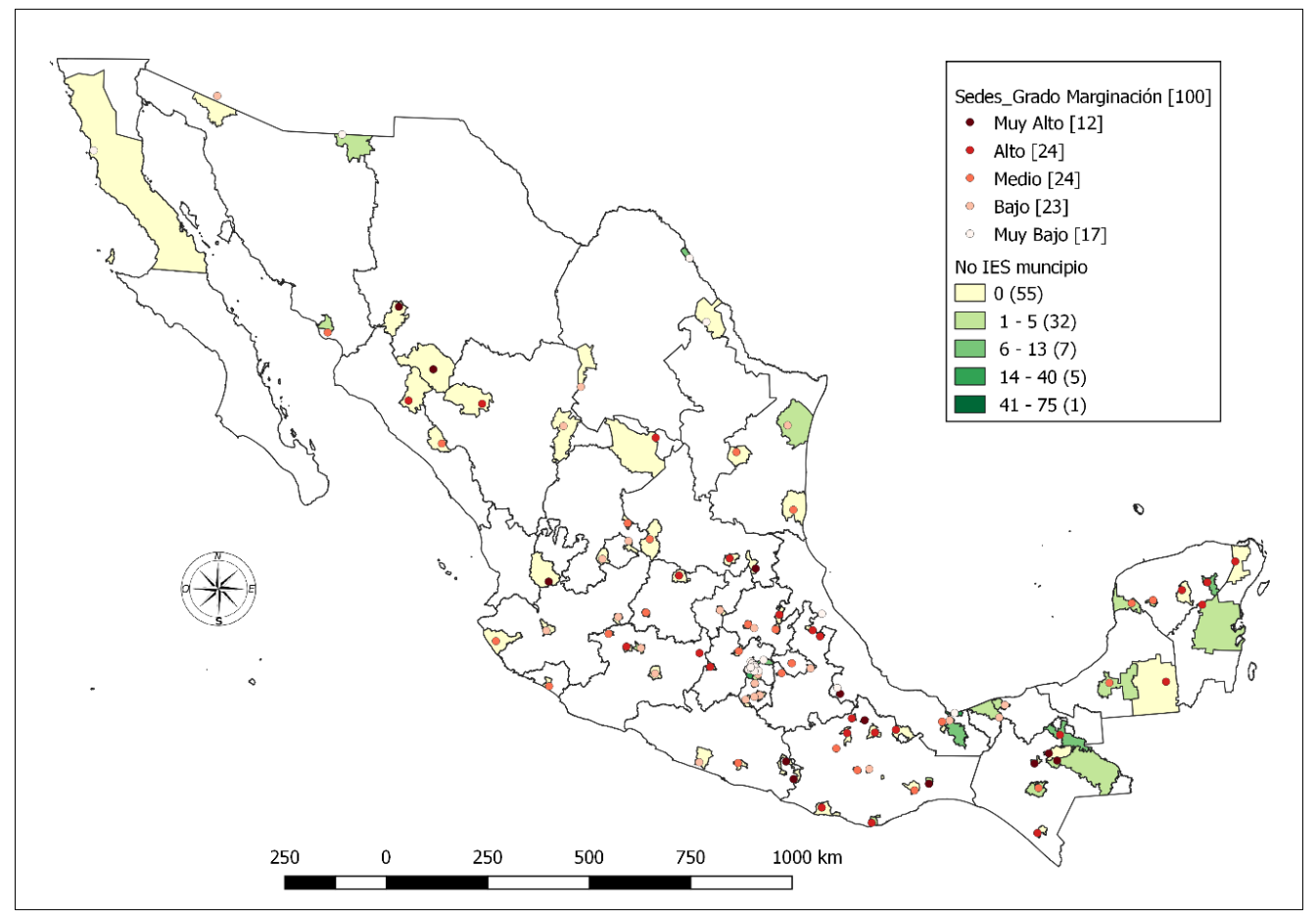

Lo primero que destaca es que el objetivo de llevar ES a los municipios pobres del país (con muy alto grado de marginación) sólo se cumple en 12 de las 100 sedes. Si agregamos a esta cifra los planteles en municipios con alto grado de marginación, el número llega a 36 sedes, de modo que este objetivo sólo se cumple en un tercio de los planteles.

De acuerdo con cifras de Asociación Nacional de Universidades e Instituciones de Educación Superior (ciclo 2017-2018), 55 de los 100 municipios no contaban con oferta de ES antes de la llegada de las UBBJG. Al parecer, el objetivo de llevar ES a donde no lo había se cumple de forma más amplia.

De nuevo, la perspectiva a nivel nacional dificulta ver qué sucede en el centro del país. Para explorar la relación entre la oferta de ES y el grado de marginación a nivel municipal en el centro del país se presenta la Figura 4. 
Figura 4. Sedes de las UBBJG por grado de marginación y oferta de ES de los municipios del centro de la República Mexicana

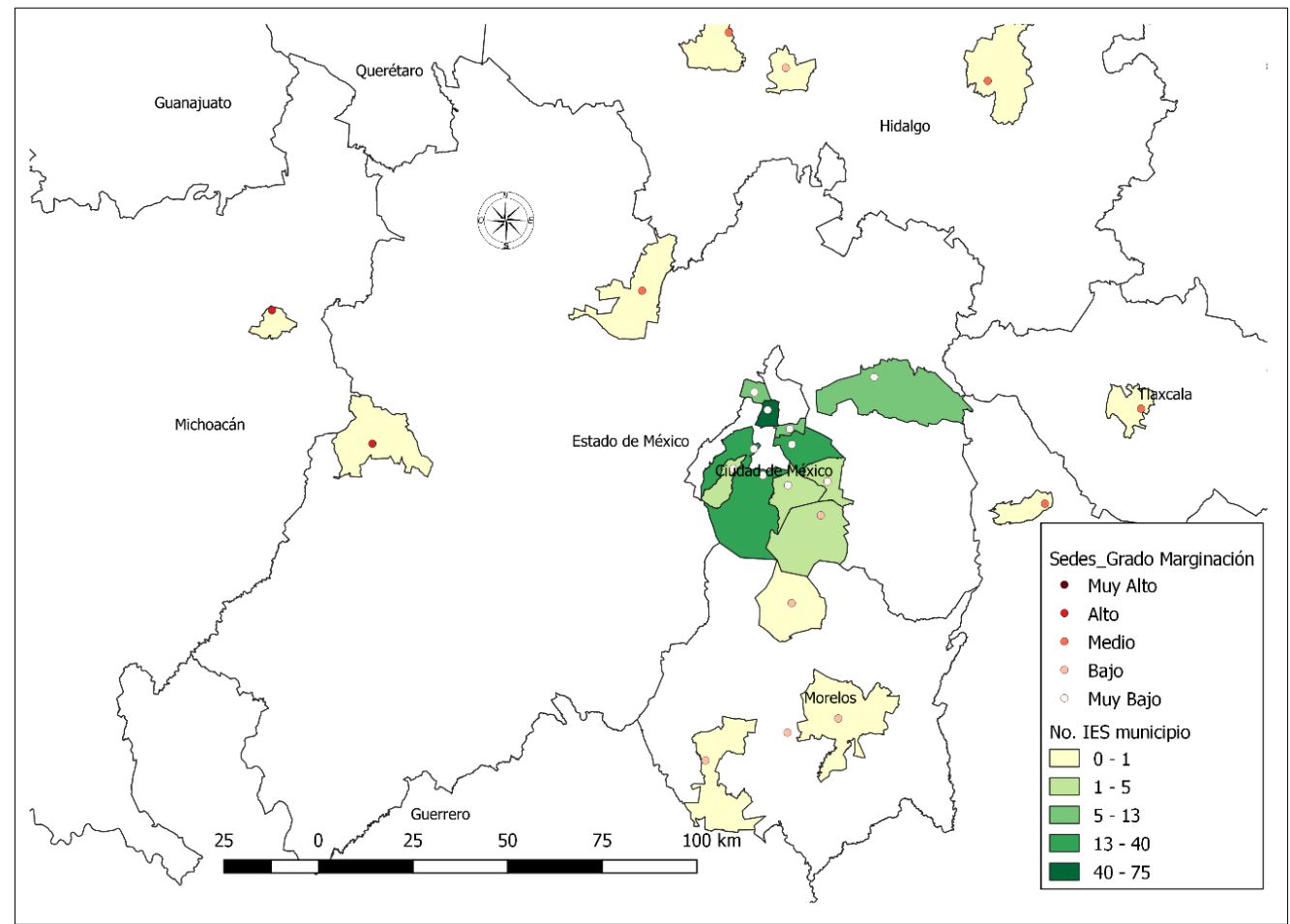

Hay al menos tres situaciones que destacar. La primera es que las sedes de las UBBJG de esta región se ubican en municipios con grados de marginación de medio a muy bajo. Si bien es cierto que las sedes allende la Ciudad de México están en municipios sin oferta de ES, las escuelas de la capital del país se localizan en alcaldías entre 5 y 75 IES. La segunda es que las sedes de las UBBJG ubicadas en la Ciudad de México contribuyen a la concentración de la oferta de ES en la parte central de la Zona Metropolitana del Valle de México, reportada previamente por Taborga (2002), Gil et al. (2009) y Mejía y González (2019); y, la tercera, es que no atiende a las poblaciones más pobres. De acuerdo con los objetivos del programa UBBJG, estas sedes no deberían ubicarse en esta región, pues no sólo no atienden a la población en condiciones de pobreza, sino que ubican una IES en una localidad que ya cuenta con ese servicio. Esta situación es doblemente grave, pues parece incrementar la tendencia a desarrollar "desiertos educativos" al interior de regiones ya de por sí económica y educativamente marginadas.

\subsection{Migración}

Рага analizar este criterio se recuperaron los datos de dos indicadores: la Intensidad Migratoria a Estados Unidos (IMEU) y la Categoría Migratoria (CM). Tales indicadores reúnen evidencia del fenómeno migratorio en sus diferentes expresiones, incorporando información de:

- Viviendas que reciben remesas (ingresos procedentes del exterior).

- Viviendas con emigrantes a Estados Unidos durante el quinquenio 2005-2010 que a la fecha del levantamiento censal permanecían en ese país (emigrantes).

- Viviendas con migrantes a Estados Unidos durante el quinquenio 2005-2010 que regresaron al país durante ese mismo periodo (migrantes circulares) y que a la fecha del levantamiento censal residían en México.

- Viviendas con migrantes que residían en Estados Unidos en 2005 y regresaron a vivir a México antes del levantamiento censal de 2010 (migrantes de retorno). 
De cada una de estas condiciones se deriva un índice que mide y diferencia la intensidad del fenómeno migratorio, tanto entre entidades federativas como entre municipios: cuanto mayor sea el índice, mayor será la intensidad de la migración en un lugar determinado (CONAPO, 2011). Por su parte, la CM refiere a cada uno de los grupos en que se puede clasificar una unidad político-administrativa de acuerdo con el rango en que se ubique su tasa neta de migración (CONAPO, s.f.).

De acuerdo con los datos del Censo 2010, de los 100 municipios sede de las UBBJG sólo 6 tienen una IMEU muy alta, 11 alta, 24 baja y 36 muy baja. Tomando como referencia este indicador, se concluye que el objetivo de ubicar a las sedes de las UBBJG en espacios de alta migración sólo se cumple en 15 municipios. En relación con la CM, 46 municipios tienen expulsión elevada. Si el indicador es la CM, el objetivo alcanza a poco menos de la mitad de los municipios. Estas cifras representan limitaciones importantes para que el programa UBBJG lleve ES a aquellas localidades con alto porcentaje de población migrante, y, en este sentido, contribuya a disminuirlo.

\subsection{Población indígena}

Para analizar en qué medida las sedes de las UBBJG se ubican en municipios con alto porcentaje de población indígena se recuperaron dos fuentes: el número de hablantes de una lengua indígena por municipio (INEGI, 2010) y la tipología de municipios en relación con su población indígena (INEGI, 2015).

Рara analizar el número de hablantes indígenas por municipio se calculó el porcentaje de este grupo en relación con la población total. Los resultados de este ejercicio se muestran en la Tabla 1. De acuerdo con sus cifras, sólo siete municipios tienen una alta concentración de personas indígenas.

Tabla 1. Distribución de los municipios sede de las UBBJG por porcentaje de hablantes de lengua indígena

\begin{tabular}{lc}
\hline Rango (porcentaje) & Número de municipios \\
\hline 0 hasta menos de 25 & 75 \\
25 hasta menos de 50 & 9 \\
50 hasta menos de 75 & 9 \\
Más de 75 & 7 \\
Total & 100 \\
\hline Nota: elaboración a partir de Censo Nacional de Vivienda 2010 (INEGI, 2010).
\end{tabular}

De acuerdo con el INEGI (2015) los municipios indígenas son aquellos con 70\% o más de población originaria. Los municipios con presencia indígena son aquellos con menos de $40 \%$ de población indígena, pero más de 5000 indígenas dentro de su población total, y con presencia importante de hablantes de lengua minoritaria. Por último, municipios con población indígena dispersa son aquellos con menos de $40 \%$ de población indígena y menos de 5000 habitantes indígenas.

Si se toma como referencia el porcentaje de hablantes de lengua indígena por municipio, sólo siete municipios sede de las UBBJG tienen 75 \% o más de población indígena. Como se muestra en la figura 5, si se toma como referencia el tipo de municipio en relación con su población indígena, sólo 26 de los 100 municipios en los que se ubican planteles de las UBBJG pueden considerarse indígenas. 
Figura 5. Tipología de municipio por porcentaje de población indígena

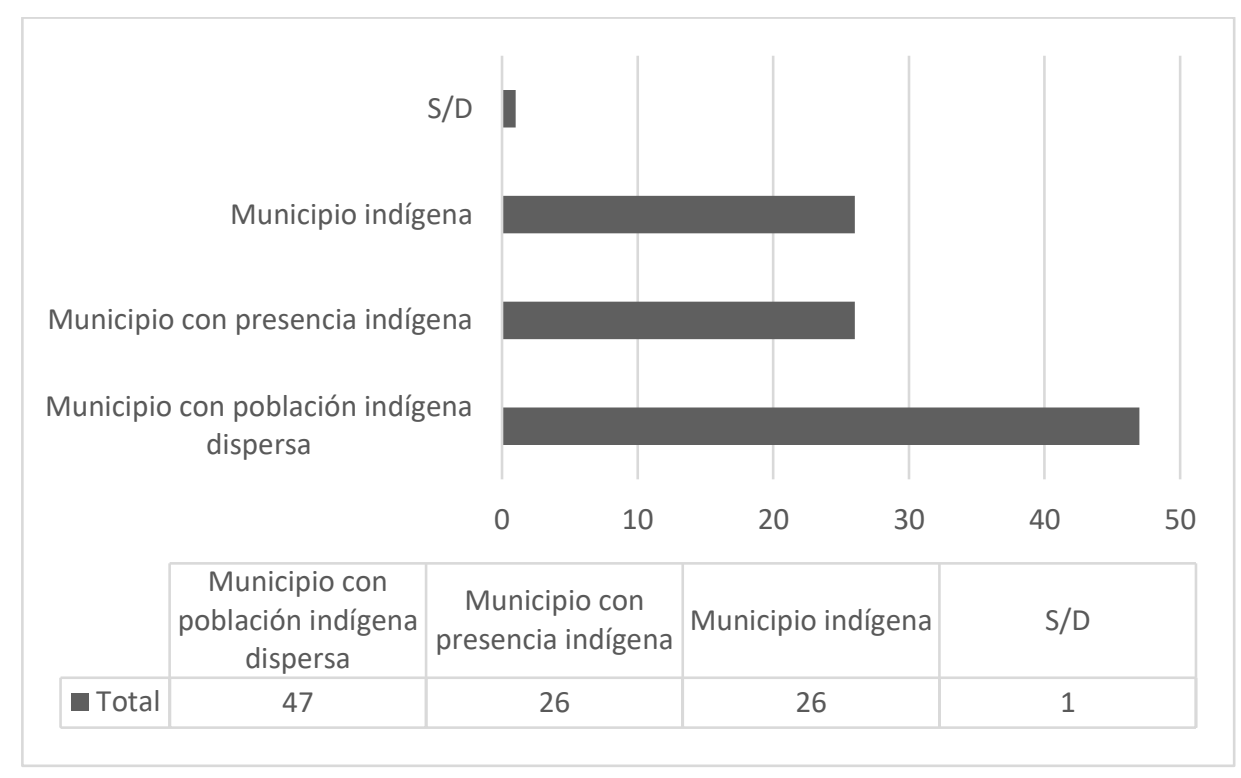

Nota: elaboración a partir de Censo Nacional de Vivienda 2010 (INEGI, 2010).

\subsection{Nivel de violencia}

Рara cerrar este análisis, se recuperaron dos indicadores para determinar el nivel de violencia en los municipios sede de las UBBJG: el Índice de Violencia Municipal (Consejo Ciudadano para la Seguridad Pública y la Justicia Penal, 2018) y la tasa de número de defunciones por homicidio (INEGI, 2015).

El Índice de Violencia Municipal sólo abarca poblaciones de 100000 o más habitantes. Dentro de este grupo sólo se localizan 21 municipios sede de las UBBJG que cumplen con el tamaño de población que toma en cuenta este instrumento. El Índice comprende los 234 municipios que tuvieron 100000 o más habitantes en 2018, según las proyecciones demográficas del CONAPO. En estos municipios ocurrieron el $71.72 \%$ de los homicidios dolosos en el país; el $66.67 \%$ de los secuestros; el $74.87 \%$ de las violaciones; el $78.04 \%$ de las lesiones dolosas; el $86.59 \%$ de los robos con violencia y el $77.66 \%$ de las extorsiones (Consejo Ciudadano para la Seguridad Pública y la Justicia Penal, 2018). El Índice considera la calificación con la base de 100 puntos y distribuye esta cantidad entre los diferentes delitos:

- 55 puntos para homicidio doloso;

- 22 puntos para secuestro;

- 13 puntos para violación;

- 4 puntos para lesiones dolosas;

- 3 puntos para robos con violencia; y

- 3 puntos para extorsión (Consejo Ciudadano para la Seguridad Pública y la Justicia Penal, 2018).

Los municipios se ordenan de manera decreciente con base en la sumatoria de sus respectivos puntajes. Entre los municipios sede de las UBBJG incluidos en el Índice, el puntaje más alto lo alcanzó la alcaldía Cuauhtémoc (Ciudad de México), con 49.79 puntos, que lo colocan en el lugar 27. El puntaje más bajo corresponde a Chilón (Chiapas), con 4.91 puntos, que lo ubican en el lugar número 229 (Consejo Ciudadano para la Seguridad Pública y la Justicia Penal, 2018).

Se calculó la tasa de homicidios (INEGI, 2015) dividiendo el número de defunciones por homicidio entre la población total por municipio, para luego multiplicar el resultado por 10000 . Se eligió esta base porque la población de los municipios sede de las UBBJG oscila entre 1815786 (Iztapalapa, Ciudad de México) y 3 218 (Áporo, Michoacán) habitantes. De los 100 municipios sede de las UBBJG, 96 tienen poblaciones de 
este tamaño (10 000 o mayor); y en el caso de los cuatro municipios que no alcanzan los 10000 habitantes, sólo se reportó una defunción por homicidio en 2015. No se eligió la base 100000 (como lo hace el Consejo Ciudadano para la Seguridad Pública y la Justicia Penal) porque sólo 21 de los 100 municipios sede tiene una población igual o mayor a esta cantidad.

Con este cálculo se determinó que el puntaje más alto fue de 37.28, obtenido por el municipio de Urique (Chihuahua) y el más bajo fue para el municipio de Valladolid (Yucatán), con 0.13. De acuerdo con este indicador, 48 municipios tienen una tasa menor a 1; 43 municipios tienen una tasa de 1 y menor a 5; y 9 tienen una tasa de 5 hasta 37.28 homicidios por cada 10000 habitantes. Si se considera como punto de corte esta última tasa para identificar los municipios más violentos, sólo nueve de los 100 municipios sede de las UBBJG están entre los más violentos del país.

Tomando en cuenta los seis criterios a partir de los cuales se eligieron los lugares sede de las UBBJG, el único municipio que cumple con ellos es Urique, en el estado de Chihuahua: tiene una población de 20386 habitantes, no tiene oferta de ES, tiene un grado de marginación muy alto, es un municipio indígena, está dentro de la categoría migratoria de expulsión elevada y tiene una tasa de homicidios de 37.2 por cada 10 000 habitantes.

\section{Discusión y conclusiones}

El acercamiento socioespacial al programa UBBJG permite entrever algunas implicaciones tanto para el cumplimiento de sus objetivos como para la política nacional de cobertura en ES.

En este texto analizamos desde una perspectiva socioespacial el cumplimiento de seis de los criterios que se tomaron en cuenta para definir la ubicación de los planteles de las UBBJG: población, pobreza, oferta de IES, migración, porcentaje de población indígena y violencia. Desde una perspectiva socioespacial las condiciones para su realización son diferenciadas. Los objetivos de acercar la oferta de ES a municipios pobres y donde se carecía del servicio resultaron ser los rubros con mejores condiciones para su cumplimiento en función de su ubicación.

Sin embargo, el análisis acumulado de las condiciones para el cumplimiento de los criterios fijados por el mismo programa en función de la ubicación de sus sedes nos muestra que sólo 52 de los 100 casos cumplen con la condición de tener entre 15000 y 50000 habitantes, si estos datos se cruzan con los de pobreza y oferta de ES, sólo siete localidades cumplen con estos criterios. La tendencia que encontramos en una versión previa de esta investigación ${ }^{2}$ fue que la incorporación de los criterios restantes disminuiría el número de municipios que cumple con los criterios establecidos en el programa. Con la posterior incorporación del criterio de que la localidad debe contar con una demanda no atendida aproximada de 200 egresados anuales de bachillerato, es probable que ninguna de las localidades cumpla con todos los criterios de selección del programa (OCUBBJG, 2020b). Esto plantea serias dudas acerca del razonamiento que está detrás de la definición de las localidades para el establecimiento de las sedes.

Más allá de las dificultades operativas que ya comienzan a presentarse (Arteta 2019a; 2019b), el propio diseño de las UBBJG muestra importantes inconsistencias de origen, pues al parecer la definición de los criterios tiene que ver más con un voluntarismo fundado en posturas ético-políticas y en los dividendos del uso político-electoral de un programa social que en criterios estrictamente educativos.

Ante este panorama incierto y cambiante surgen dudas: ¿en qué medida acercar IES a los lugares más desfavorecidos incrementará la equidad educativa?, ¿quiénes ingresarán a las UBBJG?, ¿cuántos terminarán la carrera?, ¿qué harán al egresar?, ¿cuáles han sido las experiencias de programas educativos similares en contextos rurales? Рara tratar de responder a estas preguntas consideramos pertinente continuar estudiando los probables alcances y limitaciones de las UBBJG a partir del análisis del caso de San Rafael,

2 Discutida los días 12 de agosto y 7 de octubre de 2019 en el seminario La problemática universitaria: miradas intergeneracionales, en el Instituto de Investigaciones sobre la Universidad y la Educación (IISUE), de la Universidad Nacional Autónoma de México (UNAM). 
Urique (Chihuahua), por ser la única sede que cumple con todos los criterios de ubicación establecidos por el programa.

A partir de lo expuesto en este trabajo, consideramos que las UBBJG no sólo enfrentarán condiciones adversas para el cumplimiento de sus objetivos debido al deficiente diseño de un programa social que se habría beneficiado de un análisis como el que aquí se presenta; sino también, para reducir la marginación educativa de la población que pretende favorecer mediante la creación de IES. Como se ha mostrado, el programa no ha focalizado adecuadamente sus esfuerzos y recursos para ampliar las oportunidades educativas en las regiones que más lo necesitan, lo cual podría tener un impacto incipiente en la disminución de la desigualdad en el acceso a la educación superior y en la expansión de este nivel educativo.

Además, las políticas públicas y los programas sociales pueden profundizar los procesos de marginación y exclusión que buscan disminuir. Los resultados de nuestra investigación parecen mostrar esta tendencia en el caso de las UBBJG. Si a los criterios analizados se suman factores como infraestructura, las condiciones laborales de la planta académica y los problemas de operación es probable que se hagan más evidentes las carencias del tipo de servicios educativos a los que accede la población más vulnerable.

\section{Referencias}

Acosta, A. (2005). La educación superior privada en México. IESALC-UNESCO. https://unesdoc.unesco.org/ark:/48223/pf0000140425

Arteta, I. (2019a, 30 de septiembre). A un mes de iniciar clases, Universidad Benito Juárez en Zacapu, Michoacán, se queda sin plantel. Animal Político. https://www.animalpolitico.com/2019/09/universidadbenito-juarez-zacapu-michoacan-sin-plantel/

Arteta, I. (2019b, 26 de septiembre). Alumnos de Universidades Benito Juárez en el Edomex denuncian malos tratos y desinterés de coordinadora. Animal Político.

https://www.animalpolitico.com/2019/09/alumnos-universidad-amlo-malos-tratos/

Asociación Nacional de Universidades e Instituciones de Educación Superior. (2019). Anuario educación superior-licenciatura 2017-2018. http://www.anuies.mx/informacion-y-servicios/informacion-estadisticade-educacion-superior/anuario-estadistico-de-educacion-superior

Brunner, J. J. (2005). Las transformaciones de la universidad pública. Revista de Sociología, (19), 31-49.

Brunner, J. J., P. Santiago, C. García Guadilla, J. Gerlach, y Velho, L. (2008). OECD reviews of tertiary education: Mexico. https://www.oecd.org/education/skills-beyond-school/37746196.pdf

Buttler, T. y Hamnett, C. (2007). The geography of education: Introduction. Urban Studies, 44(7), 1161-1174. https://doi.org/10.1080/00420980701329174

Carrera de beisbol, en nueve campus de las 100 universidades de López Obrador. (2019, 28 de febrero). La crónica de hoy.

https://www.cronica.com.mx/notas-

carrera de beisbol en nueve campus de las 100 universidades de lopez obrador-1111699-

2019.html

Consejo Ciudadano para la Seguridad Pública y la Justicia Penal. (2018). La violencia en los municipios de México 2018. http://seguridadjusticiaypaz.org.mx/files/La-violencia-en-los-municipios-de-Me\%CC\%81xico2018.pdf

Consejo Nacional de Población (s.f.). Glosario de Migración Interna.

http://www.conapo.gob.mx/en/CONAPO/Glosario Migracion Interna?page=1 
Consejo Nacional de Población. (2015). Índice de marginación por entidad federativa y municipio 2015. https://www.gob.mx/conapo/documentos/indice-de-marginacion-por-entidad-federativa-y-municipio$\underline{2015}$

Decreto por el que se crea el organismo público descentralizado denominado Organismo Coordinador de las Universidades para el Bienestar Benito Juárez García. Diario Oficial de la Federación [D.O.F.], 30 de julio de 2019. https://www.dof.gob.mx/nota to doc.php?codnota=5566760

Didou, S. (1997). Descentralización y urbanización del sistema de educación superior: un estado del arte y algunos hechos concretos. Revista Mexicana de Investigación Educativa, 2(3), 31-44.

https://www.comie.org.mx/revista/v2018/rmie/index.php/nrmie/article/view/1055

Didou, S. (2011). La promoción de la equidad en la educación superior en México: declinaciones múltiples. Reencuentro, (61), 7-18. https://reencuentro.xoc.uam.mx/index.php/reencuentro/article/view/769

Gil, M., Mendoza, J., Rodríguez, R. y Pérez, M. J. (2009). Cobertura de la Educación Superior en México. Tendencias, retos y perspectivas. ANUIES.

Gobierno de AMLO creará programa para abrir 100 universidades. (2018, 29 de octubre). López-Dóriga Digital.

https://lopezdoriga.com/nacional/gobierno-de-amlo-creara-programa-para-abrir-100-universidades/

Gobierno de México (2019). Sistema de Universidades Benito Juárez, desde Aquismón, San Luis Potosí. https://www.youtube.com/watch?v=WnYur Zuu8Myt=147s

Instituto Nacional de Estadística y Geografía. (2015). Encuesta Intercensal 2015.

https://www.inegi.org.mx/programas/intercensal/2015/default.html\#Tabulados

Instituto Nacional de Estadística y Geografía. (2010). Censo de Población y Vivienda 2010.

https://www.inegi.org.mx/programas/ccpv/2010/

Instituto Nacional de Estadística y Geografía. (2017). Marco Geoestadístico Nacional. https://www.ineqi.org.mx/temas/mg/default.html\#Descargas

Maldonado A. y Mejía G. (2018). Higher Education Systems and Institutions, Mexico. En P. Teixeira y J. Shin (Eds.), Encyclopedia of international higher education systems and institutions. Springer.

Márquez, A. (2004). Calidad de la educación superior en México. ¿Es posible un sistema eficiente, eficaz y equitativo? Las políticas de financiamiento de la educación superior en los noventa. Revista Mexicana de Investigación Educativa, 9(21), 477-500.

https://www.comie.org.mx/revista/v2018/rmie/index.php/nrmie/article/view/856

Mejía, G. y González, J. L. (2019). Territorios desiguales: el caso de la Universidad Mexiquense del Bicentenario. Revista Interamericana de Educación de Adultos, 41(1), 165-189.

https://www.crefal.org/rieda/images/rieda-2019-1/investigacion7.pdf

Mendoza, J. (2015). Ampliación de la oferta de educación superior en México y creación de instituciones públicas en el período 2001-2012. Revista Iberoamericana de Educación Superior, 6(16), 3-32.

https://doi.org/10.22201/iisue.20072872e.2015.16.149

Mexicanos Contra la Corrupción e Impunidad. (2020). Evaluación del programa de Universidades para el Bienestar Benito Juárez García. https://contralacorrupcion.mx/universidades-benito-juarez-en-el-limbo/

Montes, M. (2018, 7 de octubre). Reitera AMLO: creará 100 universidades; 5 en Michoacán. Transmedia 
Televisión. http://www.transmediatelevision.com/reitera-amlo-creara-100-universidades-5-en-michoacan/

Organismo Coordinador de las Universidades para el Bienestar Benito Juárez García. (2020a). Estatuto Académico. https://ubbj.gob.mx/registro\#inicio

Organismo Coordinador de las Universidades para el Bienestar Benito Juárez García. (2020b). Lineamientos para la instalación/rehabilitación y equipamiento de las sedes educativas del Programa Universidades para el Bienestar Benito Juárez García en el Programa Presupuestal U083, correspondiente al OCUBBJG. https://ubbj.gob.mx/registro\#inicio

Poder Ejecutivo Federal. (2007). Plan Nacional de Desarrollo 2007-2012.

https://paot.org.mx/centro/programas/federal/07/pnd07-12.pdf

Poy, L. (2018, 20 de diciembre). Para indígenas y los más pobres, las cien nuevas universidades. La Jornada. https://www.jornada.com.mx/ultimas/2018/12/28/para-indigenas-y-los-mas-pobres-las-cien-nuevasuniversidades-4108.html

Roldán, N. (2019, 20 de marzo). Sin planteles construidos, este mes arrancan las 100 nuevas universidades de AMLO. Animal Político. https://www.animalpolitico.com/2019/03/100-nuevas-universidades-amlobenito-juarez/

Salazar, A. (2020, 5 de enero). En 2021, los primeros titulados de las universidades de la 4T. El Heraldo. https://heraldodemexico.com.mx/pais/2021-primeros-titulados-universidades-4t-bienestar-amloplanteles/

Secretaría de Educación Pública. (2018). Sistema Educativo de los Estados Unidos Mexicanos. Principales cifras 2017-2018.

https://www.planeacion.sep.gob.mx/Doc/estadistica e indicadores/principales cifras/flash/index.html

Secretaría de Educación Pública. (2019). Programa de Universidades para el Bienestar Benito Juárez García. https://www.gob.mx/cms/uploads/attachment/file/465578/Programa de Universidades para el Bienes $\underline{\operatorname{tar} B J . p d f}$

Secretaría de Hacienda y Crédito Público. (2019). Presupuesto de Egresos de la Federación. Ejercicio Fiscal 2020. https://www.pef.hacienda.gob.mx/es/PEF2020/home

Silas, J. (2005). Realidades y tendencias en la educación superior privada mexicana. Perfiles Educativos, 27(109-110), 7-37.

Silva, M. (2014). Equidad en la educación superior mexicana: el reto persistente. Universidades, (59), 23-25. https://www.redalyc.org/articulo.oa?id=37332547004

Socio-Economic Database for Latin America and the Caribbean y Banco Mundial (2017). Estadísticas. http://www.cedlas.econo.unlp.edu.ar/wp/estadisticas/sedlac/estadisticas/\#1496165425791-920f2d43-

$\underline{\text { f84a }}$

Taborga, H. (2002). Oferta y demanda de estudios de licenciatura en la Zona Metropolitana de la Ciudad de México. ANUIES.

Taylor, C. (2009). Towards a geography of education. Oxford Review of Education, 35(5), 651-669. https://www.jstor.org/stable/27784591

Universidades para el Bienestar Benito Juárez García. (2020). Portal oficial de las Universidades para el Bienestar Benito Juárez García. https://ubbj.gob.mx/ 